Le purpura vélo-palatin « a vacuo » de Barthélemy (1928) pÿrevisité : Barthélemy s a vacuo palatal purpura (1928) revisited

Kluger, $\mathrm{N}$.

2017

Kluger , N 2017 , ' Le purpura vélo-palatin « a vacuo » de Barthélemy (1928) revisité :

pÿBarthélemy s a vacuo palatal purpura (1928) revisited ', Annales de Dermatologie et de Vénéréologie , vol. 144 , no. 1 , pp. 81-83 . https://doi.org/10.1016/j.annder.2016.07.014

http://hdl.handle.net/10138/231681

https://doi.org/10.1016/j.annder.2016.07.014

publishedVersion

Downloaded from Helda, University of Helsinki institutional repository.

This is an electronic reprint of the original article.

This reprint may differ from the original in pagination and typographic detail.

Please cite the original version. 


\title{
Le purpura vélo-palatin «a vacuo » de Barthélemy (1928) revisité
}

\section{Barthélemy's "a vacuo" palatal purpura (1928) revisited}

\author{
N. Kluger
}

University of Helsinki, Helsinki university central hospital: dermatology and allergology, Meilahdentie 2, PO Box 160, 00029 Hus, Finlande

Reçu le 22 mai 2016 ; accepté le 26 juillet 2016

Disponible sur Internet le 15 septembre 2016

En 1928, les Annales de maladies vénériennes (publiées en France de 1906 à 1940) rapportent un cas clinique succin, mais des plus inhabituels, par Monsieur le Dr Barthélemy [1]. Inhabituel en raison de la cause identifiée, qui peut faire sourire, mais également par la truculence et l'enthousiasme dont fait preuve le narrateur.

Ainsi, le Dr Barthélémy ${ }^{1}$ rapporte un cas, datant déjà, d'il y a «quelques années », d'une femme examinée dans le cadre d'une visite sanitaire dans une "maison » (maison est imprimée entre guillemets dans le texte). Lors de l'examen de la bouche de la patiente, dont ni l'âge, ni les antécédents ne nous sont donnés, le médecin identifie de «multiples taches purpuriques dont certaines atteignent le diamètre d'une pièce de $0 \mathrm{fr} 50$ ». Elles sont « grossièrement arrondies, un peu irrégulières sur le contour, (...), franchement ecchymotiques, sur un fond muqueux normal ». Le reste de l'examen bucco-pharyngé et l'examen clinique général de cette patiente sont strictement normaux. Aucune autre lésion purpurique cutanée ou muqueuse n'est trouvée. Le médecin reste perplexe devant ce «purpura vélo-palatin indolent », que la patiente n'avait d'ailleurs pas remarqué

\footnotetext{
Adresse e-mail : nicolas.kluger@hus.fi

${ }^{1}$ Le prénom et le lieu d'activité du Dr Barthélemy ne sont pas précisés dans l'article.
}

et pour lequel il élimine toutes les causes locales possibles (affection dentaire, médicament, brûlure par aliment, boisson, condiments ou cigarettes ainsi que l'empalement traumatique par le crayon tenu droit en bouche).

Si la première partie du texte est rédigée comme toute observation clinique de l'époque avec force de détails cliniques, le paragraphe relatant la cause des symptômes semble sortir tout droit de la Philosophie dans le boudoir ou de quelques autres extraits de la littérature érotique. En effet, devant la perplexité du médecin, c'est la patiente elle-même qui suggère le diagnostic étiologique en se remémorant « un client de l'autre jour » (confirmant de fait, ce non-dit évident pour le lecteur de l'époque, à savoir qu'il s'agit d'une prostituée et d'une visite au sein d'une « maison » close). On apprend que ce client était «plein d'exigences ». Ce dernier avait « non seulement demandé le coït $a b$ ore, mais il avait réclamé de la conviction, de l'ardeur, de l'enthousiasme, de la frénésie, du délire bachique ». La patiente, «partenaire consciencieuse » (...) «s'était attachée au travail comme la pieuvre à sa proie ». Par «orgueil professionnel », « elle avait mis tout son zèle, tout son ambition dans la réussite, et ses persévérantes aspirations avaient fini par obtenir un succès triomphal ». Pour Barthélemy, les lésions purpuriques s'expliquent par un «vide aussi poussé » qui aurait créé une « dépression barométrique telle, que des suffusions sanguines s'étaient 
produites au point le plus exposé et le plus fragile (...)». Barthélemy rapporte dans la foulée un second cas rencontré l'année suivante pour lequel, fort de son expérience, il évoque immédiatement le diagnostic et fait «avouer à cette seconde les sévices pneumatiques qu'elle avait subis. . . activement $»$.

Barthélemy décrit ici avec force d'images fleuries mais ô combien parlantes, les deux premiers cas de purpura palatin induit par fellation [1].

Il faudra attendre environ 20 ans pour que le $1^{\text {er }}$ cas soit rapporté en langue anglaise par Rattner, en 1949, qui relate le cas d'une femme qui présentait des lésions récidivantes palatines uniquement lors de ses menstruations car cette dernière " pratiquait l'art » uniquement à ces périodes [2]. Par la suite, les publications fleurissent dans les années 1975-1980 [3-7] et puis connaissent un hiatus notable d'une vingtaine d'années, hormis quelques publications éparses $[8,9]$. Les dernières publications sur le sujet datent de 2013 [10-12]. À notre connaissance, le nombre de publications dans la littérature avoisine environ la quinzaine. Nous n'avons pas trouvé de mention de cette « entité » dans le traité de Degos, que ce soit dans le chapitre purpura ou dans celui dédié à la muqueuse buccale.

Depuis la description princeps de Barthélemy, la présentation clinique reste assez stéréotypée. Il s'agit le plus souvent de jeunes femmes en bonne santé, plus rarement d'hommes ou des patientes d'âge mur [4], se présentant avec des lésions asymptomatiques, de découverte fortuite le matin lors du brossage ou de l'utilisation de fil dentaire [11] ou bien lors d'un examen bucco-dentaire chez le médecin ou le dentiste. On observe un érythème palatin, un purpura pétéchial ou ecchymotique sur le palais mou, le palais dur ou à la jonction, épargnant en revanche l'uvule et le mur pharyngé postérieur (Fig. 1). Comme mentionné plus haut, une récidive à certains intervalles des symptômes est possible selon les habitudes de la pratique (fellation en période menstruelle etc.) [2]. Les lésions disparaissent habituellement dans les jours qui suivent sans séquelles. Si une biopsie était pratiquée, elle montrerait une hémorragie au niveau de la lamina propria, éventuellement un décollement vésiculeux hémorragique sous-épithélial et un infiltrat de leucocytes et d'érythrocytes au sein de l'épithélium [7]. Dans un cas, une mucite palatine localisée à Candida albicans était diagnostiquée chez une femme avec une candidose vaginale et dont le partenaire avait une candidose pénienne [7]. Il s'agit là d'un diagnostic différent de celui du purpura de Barthélemy.

Une liste non exhaustive des diagnostics différentiels des purpuras palatins est résumée dans le Tableau 1. Une fois le diagnostic étiologique établi par l'anamnèse, aucun examen complémentaire, ni traitement ne sont nécessaires. En l'absence de prise de risque sexuel, il ne semble pas licite de proposer un dépistage des infections vénériennes. Une abstinence « orale » temporaire peut être suggérée tout au plus, mais n'est en rien indispensable car ces lésions peuvent régresser malgré le maintien de la pratique [12].

Alors que presque $80 \%$ des hommes et femmes admettent pratiquer (ou recevoir) le sexe oral [13], on peut s'étonner de prime abord du faible nombre de cas rapportés. S'agit-il d'une entité au final "classique », à ce point connue de tous les médecins qu'elle n'est plus rapportée ? Ou au contraire est-elle méconnue et non diagnostiquée par ces derniers ?

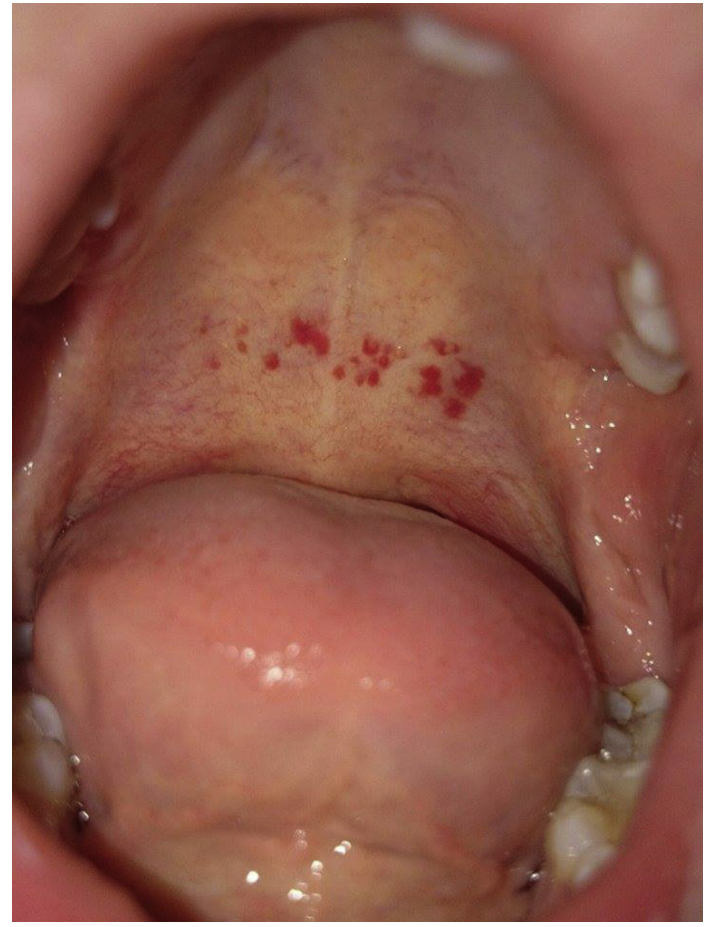

Figure 1. Purpura pétéchial palatin résolutif chez une jeune femme de 20 ans (collection Dr Korkiavuori, Harjavalta, Finlande).

Tableau 1 Diagnostics différentiels du purpura palatin (non exhaustif, modifié d'après Cohen et Miller [11]).

Troubles de l'hémostase et de la coagulation

Thrombopathie acquise ou constitutionnelle Thrombopénie acquise ou constitutionnelle Médicaments : antiagrégants plaquettaires, anticoagulation

Infection

Mononucléose infectieuse

Infections streptococciques et autres infections des voies respiratoires

Traumatismes

Empalement direct d'un objet (crayon, sucette, sonde d'intubation, sonde naso-gastrique, etc.)

Effort violent de toux, d'éternuement ou de vomissements ; manœuvre de Heimlich

Réflexe de frottement répété de la langue sur le palais (prurit palatin allergique, bijou de piercing de langue)

Brûlure chimique

Brûlure thermique

Pratique d'un instrument à vent ou d'un cuivre

Succion

Fellation

Maladies systémiques

Carence en vitamine C (scorbut)

Amylose

Autres causes locales

Angine bulleuse hémorragique 
La chronologie des événements et de la consultation est évidemment fondamentale car elle doit avoir lieu le lendemain ou les quelques jours suivant le rapport oral. De plus, les patients qui consultent ne font probablement pas toujours eux-mêmes le lien. Certain(e)s peuvent bien évidemment être réticent(e)s à s'épancher sur leur sexualité, notamment s'il s'agit de jeunes patient(e)s, accompagné(e)s des parents, ou d'hommes mariés [7]. Le médecin lui-même peut rechigner à poser ce type de question par peur d'être inconvenant ou intrusif. Par ailleurs, il faut éviter les jugements hâtifs et toujours garder ce diagnostic en tête, quel que soit le ou la consultante. Ainsi, Giansanti et al. reconnaissaient ne pas avoir considéré ce diagnostic possible pour des « raisons évidentes » chez une femme de 56 ans obèse ( « the etiologic factor responsible for Cases 1 and 2 was not considered for what were thought to be obvious reasons ») [4]. Fort heureusement, certains patients finissent cependant par suggérer cette possibilité devant la perplexité du médecin à trouver la cause des ces lésions. Rappelons que, malheureusement, ce même purpura palatin, de découverte fortuite, chez un enfant doit faire considérer un possible abus sexuel [14].

Sur le plan physio-pathogénique, pour Barthélemy [1] et la plupart des auteurs [2,11], les lésions sont le résultat de la pression négative appliquée sur la partie postérieure de l'oro-pharynx lors de la mise en action des muscles tenseur et élévateur du voile du palais durant la fellation. On peut émettre quelques doutes sur cette hypothèse et sur l'importance du vide barométrique créée lors de la fellation pour aboutir à telles lésions chez l'officiant(e). D'autres se sont hasardés à proposer, tout simplement, le contact direct du gland du pénis qui frappe avec plus ou moins de force le palais lors de l'acte [12].

Le purpura vélo-palatin après fellation existe et est probablement sous-estimé ou sous-diagnostiqué. Il peut toucher n'importe qui, à n'importe quel âge. Il convient de le connaître pour rassurer les patients les plus anxieux et éviter les explorations inutiles. Toutes les fellations ne s'accompagnent pas de ce type de lésions car il faut un acte actif, vigoureux et (peut-être ?) prolongé. Au final, depuis Barthélemy, on n'a pas fait mieux en termes de description physio-pathogénique.

\section{Déclaration de liens d'intérêts}

L'auteur déclare ne pas avoir de liens d'intérêts.

\section{Références}

[1] Barthelemy. Une affection vénérienne peu banale : le purpura vélo-palatin «a vacuo». Ann Mal Vener 1928;23:451-3.

[2] Rattner H. A strange case of palatitis. Arch Derm Syphilol 1949;60:624.

[3] Schlesinger SL, Borbotsina J, O’Neill L. Petechial hemorrhages of the soft palate secondary to fellatio. Oral Surg Oral Med Oral Pathol 1975;40:376-8.

[4] Giansanti JS, Cramer JR, Weathers DR. Palatal erythema: another etiologic factor. Oral Surg Oral Med Oral Pathol 1975;40:379-81.

[5] Worsaae N, Wanscher B. Oral injury caused by fellatio. Acta Derm Venereol 1978;58:187-8.

[6] Bellizzi R, Krakow AM, Plack W. Soft palate trauma associated with fellatio: case report. Mil Med 1980;145:787-8.

[7] Damm DD, White DK, Brinker CM. Variations of palatal erythema secondary to fellatio. Oral Surg Oral Med Oral Pathol 1981;52:417-21.

[8] Aloi FG. Suffused ecchymosis of the palate caused by fellatio. G Ital Dermatol Venereol 1984;119:351-2.

[9] Luotio K. Oral sex causing palatal mucosal erythema. Case description and review of the differential diagnosis. Suom Hammaslaakarilehti 1986;33:1226-8.

[10] Terezhalmy GT, Riley CK, Moore WS. Oral lesions secondary to fellatio. Quintessence Int 2000;31:361.

[11] Cohen PR, Miller VM. Fellatio-associated petechiae of the palate: report of purpuric palatal lesions developing after oral sex. Dermatol Online J 2013;19:18963.

[12] Oliveira SC, Slot DE, Van der Weijden GA. What is the cause of palate lesions? A case report. Int J Dent Hyg 2013;11:306-9.

[13] Stone N, Hatherall B, Ingham R, McEachran J. Oral sex and condom use among young people in the United Kingdom. Perspect Sex Reprod Health 2006;38:6-12.

[14] Arora R, Hartwig E, Kannikeswaran N. Oral lesion secondary to child abuse. J Emerg Med 2013;45:e139-40. 